\title{
Tendencias recientes de la migración internacional y las remesas en Coahuila
}

Resumen: El propósito fundamental de este trabajo es ofrecer un diagnóstico de la situación que presenta hoy en día la migración internacional de coahuilenses hacia los Estados Unidos. En particular, se pretende valorar el fenómeno en tres vertientes: 1) con base en su importancia relativa dentro del contexto nacional; 2) en referencia a la intensidad y dinámica del fenómeno, y 3) en función de su trascendencia económica.

Palabras clave: migración, remesas, pobreza, patrón migratorio, ingreso.

Abstract This essay examines continuities and changes in the profile of Coahuilenses migration to United States and reflects on that question in an attempt to cast some light on: (a) the economic and social importance of Coahuilenses migration to the USA, (b) the main qual-

\footnotetext{
* Profesor-Investigador del Centro de Análisis y Evaluación de la Política Pública, Escuela de Graduados en Administración Pública y Política Pública del Instituto Tecnológico de Monterrey. Se le puede enviar correspondencia a Av. Eugenio Garza Sada 2501 Sur, Edif. CEDEs, 99 piso, oficina 906, C. P. 64849, Monterrey, Nuevo León. Correo electrónico: hrr@ itesm.mx
} 
itative changes and problems associated with it, and (c) the role played by remittances for help to diminish poverty level.

Key words: migration, remittances, poverty, migratory pattern, income.

\section{Introducción}

La migración de mexicanos hacia los Estados Unidos es un fenómeno con una larga historia en nuestro país. Ha venido adquiriendo proporciones masivas en décadas recientes, con singulares repercusiones socioeconómicas, políticas y culturales en ambos lados de la frontera. La gran mayoría de los flujos migratorios hacia el vecino del norte han provenido tradicionalmente del occidente y centro-norte de México, en particular de los estados de Jalisco, Michoacán, Guanajuato, Zacatecas, Durango, San Luis Potosí y, en menor medida, Colima y Aguascalientes ${ }^{1}$ Sin embar- $^{-}$ go, en los últimos años otras poblaciones se han sumado al flujo internacional, que junto con los cambios registrados en el espectro ocupacional de los trabajadores mexicanos en los Estados Unidos, los cambios en los patrones migratorios tradicionales (en términos de edad, sexo, escolaridad, posición en el hogar, tiempo de estancia, etcétera) y el monto, usos e impactos de las remesas familiares, han venido a configurar un nuevo escenario de la migración internacional de mexicanos hacia los Estados Unidos.

En las últimas décadas, los estudios sobre el fenómeno han producido información empírica valiosa y propuesto vetas teóricas de gran riqueza para su comprensión. No obstante esto, la

1 Jorge Durand caracteriza a estos estados como "la región histórica de la migra ción mexicana hacia los Estados Unidos". Para mayor información, consultar Durand (1998). 
diversificación y complejidad que ha venido registrando el flujo migratorio hacia el vecino del norte hace necesario extender el análisis hacia nuevos espacios regionales en los que las especificidades socioeconómicas, culturales y geográficas están determinando las formas diversas en que se presenta el fenómeno.

En este contexto, el propósito central de este trabajo es ofrecer un diagnóstico de la situación que presenta hoy en día la migra ción internacional de coahuilenses hacia los Estados Unidos. En particular, se pretende valorar el fenómeno en tres vertientes. 1) con base en su importancia relativa dentro del contexto nacional; 2) en referencia a la intensidad y dinámica del fenómeno, y 3) en función a su trascendencia económica. La hipótesis fundamental que guía el desarrollo de la presente investigación está orientada a demostrar que si bien en términos cuantitativos la migra ción internacional de coahuilenses hacia los Estados U nidos no es muy significativa como en otras entidades del país, la funcionalidad económica del fenómeno es de suma trascendencia para el sostenimiento de las familias y la reducción de los niveles de pobreza.

La fuente principal de información estadística que se utiliza en esta investigación proviene de cálculos propios obtenidos a través de las bases de datos del Conteo General de Población y Vivienda de 1995 y el xII Censo General de Población y Vivienda del año 2000, realizado por el INEGI.

El trabajo se encuentra estructurado en cuatro secciones, aparte de esta introducción y unas reflexiones finales La primera de ellas presenta un panorama general de la situación actual que guarda la migración de mexicanos hacia los Estados Unidos; la segunda sección ubica la importancia relativa que tiene Coahuila en el flujo de connacionales hacia el vecino del norte; por su parte, la tercera trata de mostrar el perfil del migrante coahuilense y su localización espacial dentro de la entidad. Finalmente, la última sección dimensiona la importancia económica que tiene la migración internacional hacia los Estados U nidos tanto en el plano nacional como en el ámbito local y familiar. 


\section{La migración mexicana hacia los Estados Unidos}

La migración permanente de mexicanos hacia los Estados Unidos tiene su origen a finales del siglo XIX, cuando en el suroeste de aquel país se emprendieron grandes obras de infraestructura encabezadas por la construcción del ferrocarril, las presas y los sistemas de riego, que generaron significativos impactos tanto en el desarrollo de la agricultura comercial y la minería de esa región como en la contratación, de manera sistemática, de mano de obra mexicana a través de los enganchadores (Bustamante, 1997). 2 Durante las primeras décadas del siglo xx, continuó el flujo de mano de obra mexicana hacia el vecino del norte, potenciada por la Revolución Mexicana de 1910-1921 y la Primera Guerra Mundial. Si bien para 1930, el Censo de Población de los Estados Unidos registró 730 mil personas nacidas en México y ra dicadas en ese país, a lo largo de esa década, y producto de la gra ve crisis por la que atravesaba la Unión Americana, tuvieron lugar repatriaciones masivas que redujeron el número de mexicanos en los Estados Unidos.

No obstante lo anterior, este proceso se detuvo a raíz del inicio de la Segunda Guerra Mundial, que no sólo produjo un gran aumento de mano de obra, sino también el primer acuerdo formal de trabajadores huéspedes entre México y Estados Unidos, signado en 1942 (Escobar, Bean y Weintraub, 1999). 3 Durante sus 22 años de duración, el acuerdo proporcionó 4.5 millones de contratos de trabajadores, y llegó a su fin en 1964. A partir de entonces, si bien los mexicanos han representado una parte impor-

\footnotetext{
2 Para algunos investigadores, la migración de connacionales hacia el vecino del norte comenzó con los mexicanos que decidieron quedarse en los territorios arreba tados por la potencia norteamericana luego de la guerra de 1847-1848; Bean y Tienda (1987) señalan que el número de mexicanos radicados en los estados que pasaron a ser territorio estadounidense en esta época fue de aproximadamente 80 mil personas.

3 El Programa Braceros, como fue conocido en México, estipulaba la migración temporal legal de mexicanos a los Estados Unidos, sobre todo para desempeñar labores agrícolas.
} 
tante de la inmigración legal a los Estados Unidos, el peso de la ilegal ha ido en aumento, y en la actualidad constituye el flujo mayoritario de población desde México.

Es evidente que en el proceso histórico de la migración de mexicanos hacia los Estados Unidos han intervenido factores diversos tales como la vecindad geográfica, los acuerdos migratorios, las voluntades políticas de ambas naciones, la evolución económica del país vecino y el difícil contexto económico del nuestro; son los que han nutrido las distintas intensidades y orientaciones del fenómeno. Sin embargo, en las últimas décadas se ha hecho ca da vez más evidente que éste es, en esencia, un fenómeno laboral que ha estado potenciado por: 1) el intenso ritmo de crecimiento demográfico de la población mexicana en edad laboral y la insuficiente dinámica de la economía nacional para ofrecer un trabajo digno y bien remunerado; 2) la persistente demanda de mano de obra mexicana en los sectores agrícola, industrial y de servicios en la Unión Americana; 3) el considerable diferencial sala rial entre ambas economías, y 4) la tradición migratoria ( redes de migración) hacia el vecino país del norte, conformada desde el siglo xIX y sobre todo durante el siglo pasado en muy diversas regiones del país 4

Las estimaciones más recientes sobre la magnitud del fenómeno revelan que:

1. El Censo de Población del año 2000 realizado en los Estados Unidos registró la existencia de 9.2 millones de personas nacidas en México y que residían de manera autorizada o no autorizada en ese país. Esta cifra equivale al $9.5 \%$ de la población total de México y 3.3\% de la de Estados Unidos. 5

4 Un análisis más amplio de estos aspectos se puede encontrar en conapo (2002a), Escobar, Bean y Weintraub (1999), así como Delgado y Rodríguez (1999).

5 Para el año 2000, los mexicanos residentes en Estados Unidos representaron el 30 por ciento de la población extranjera en ese pás (cuyo monto asciende a $31.2 \mathrm{mi}$ llones de personas) y el 58 por ciento de los migrantes latinos. 
2. Para dar una idea más clara de la intensidad que ha tenido la migración internacional mexicana hacia aquel país, esimportante anotar que de 1960 al año 2000, la cifra de connaciona les residentes en el vecino del norte se incrementó 16 veces al pasar de poco más de medio millón de personas a los $9.2 \mathrm{mi}$ llones, ya antes mencionados, y se convirtió así en el grupo de inmigrantes hacia los Estados Unidos con mayor dinamismo en términos de su crecimiento. ${ }^{6}$

3. El dinamismo migratorio se concentró significativamente en la década pasada, pues entre 1990 y el año 2000, el número de mexicanos que se fueron a vivir a los Estados Unidos fue de ca si 4.9 millones de personas, cifra que representa el 5.2 por ciento de la población total mexicana para el año 2000. S a la población total de residentes nacidos en México se suman los estadounidenses de origen mexicano, es posible afirmar que en Estados Unidos se encuentran establecidos casi 23 millones de personas con estrechos vínculos consanguíneos con nuestro país (CONAPO, 2002a).

Más allá de estas cifras cuantitativas, que de suyo ponen de relieve la magnitud alcanzada por el fenómeno de la migración internacional, hay una serie de cambios cualitativos que han venido a modificar la imagen tradicional de los emigrantes mexicanos, vigente hasta los años sesenta. Por aquellos años, el citado proceso se caracterizaba por ser un flujo predominante circular (de ida y vuelta), compuesto por adultos y jóvenes de origen rural que procedían de siete u ocho entidades federativas del país y cuya principal ocupación en el mercado laboral norteamericano era la de trabajadores agrícolas. Todo este proceso derivó en una migración de carácter temporal con estancias entre seis y siete meses (Escobar, Bean y Weintraub, 1999:29).

\footnotetext{
6 Para 1970, asciende a 759 mil residentes mexicanos en ese pás; en 1980, se ubica en 2.2 millones de personas y ya para 1990, alcanza la significativa cifra de 4.3 millones de mexicanos Información obtenida de la página electrónica del U. S.Census Bureau (http:// www.census.gov/ main/ www/ cen2000.html).
} 
Por el contrario, el flujo migratorio actual manifiesta un patrón más complejo y heterogéneo (Delgado y Rodríguez, 2000:374):

1. Una estancia más larga de los migrantes en el vecino país del norte o bien en el establecimiento de su residencia permanente en Estados Unidos. Por ello es evidente un cambio significativo del patrón tradicional de migración;

2. Una creciente diversificación regional del flujo que se ha extendido más allá de las entidades tradicionales de emigración. ${ }^{7}$ Actualmente se originan cuantiosas corrientes migratorias en entidades que en el pasado no se contaban entre las de tradición migratoria, como Morelos, Puebla,Hidalgo, el Estado de México y el Distrito Federal;

3. Una cada vez más notoria presencia de los migrantes procedentes de las zonas urbanas; y

4. Una mayor diversificación ocupacional y sectorial de los migrantes tanto en México como en Estados Unidos. En la actualidad, los migrantes que desempeñan una ocupación agrícola ya no son mayoritarios ni en su lugar de origen ni en el de destino.

Cabe señalar que se ha generado, en años recientes, un intenso debate entre aquellos que sostienen una continuidad del pa trón migratorio de mexicanos hacia los Estados Unidos y otros que argumentan aspectos significativos de cambio en la dinámica migratoria mexicana. Entre los primeros destacan Durand, Massey y Zenteno (2001), que con datos provenientes de la Encuesta Nacional de la Dinámica Demográfica (ENADID-1997) y del Mexican Migration Project, tratan de mostrar la estabilidad en el patrón migratorio de los mexicanos hacia el vecino del norte, enfatizando la idea de que el fenómeno de la migración sigue sien-

\footnotetext{
7 Esto no significa que en dichas áreas haya tendido a disminuir el flujo, sino que se incrementó en otras.
} 
do dominado por los estados tradicionalmente expulsores (Jalisco, Guanajuato, Michoacán y Zacatecas) y con un alto componente de participación masculina joven en edad laboral.

Por el otro, cobra singular importancia el trabajo realizado por Marcelli (2001), quien, con información derivada del Censo de Población y Vivienda del año 2000, realizado en México, y la Cu rrent Population Survey (CPS), de los Estados Unidos, encuentra relevantes transformaciones del patrón migratorio tradicional, entre las que destacan la amplia diversificación geográfica del lugar de origen de los migrantes mexicanos, una creciente participación de la mujer en el flujo y una tendencia a mayor tiempo de estancia de los migrantes mexicanos en el vecino del norte.

La información estadística que se ha obtenido en esta investigación concuerda más con aquellos trabajos que la segunda hipótesis, es decir, la de un cambio en el patrón migratorio.

\section{Coahuila en $\mathrm{e}$ contexto de la migración intemacional mexicana hacia los Estados Unidos}

Para poder entender la migración de coahuilenses hacia los Estados Unidos y ubicar en su justa dimensión la importancia del fenómeno dentro del concierto nacional, es necesario delinear algunos indicadores macroeconómicos y sociales básicos que permitan valorar las condiciones estructurales de la entidad y el contexto en el cual se desarrolla la emigración hacia el país vecino. Hay cuatro datos socioeconómicos que resultan ser de gran trascendencia a este respecto:

Primero, a diferencia de las tendencias oscilantes que caracterizaron el desempeño de la economía mexicana a lo largo de la década pasada, Coahuila se situó como una de las entidades más sólidas y estables en términos de su crecimiento económico. Las estadísticas oficiales publicadas por el INEGI revelan que entre 
1993 y el año 2000 el crecimiento del PIB de la entidad fue del orden de $\mathbf{5 . 3}$ por ciento promedio anual contra 3.9 por ciento registrado en el plano nacional. Destaca de manera singular la dinámica mostrada por la industria manufacturera; transporte y comunicaciones, y el sector de electricidad, gas y agua.

Segundo, entre 1990 y el año 2000 el número de ocupaciones generadas en el estado fue de casi 260 mil nuevas plazas, similar a una tasa de generación de empleos de $4.4 \%$ anual. Dato que superó con poco más del doble al registrado por el de la población en edad activa, pues su tasa de crecimiento se ubicó en el orden de $\mathbf{1 . 9 \%}$ de crecimiento promedio anual durante el citado periodo.

Tercero, en términos de hogares pobres, Coahuila se caracteriza por reflejar un promedio inferior al nacional. Cálculos propios, con datos obtenidos de la base de datos del xII Censo General de Población y Vivienda del año 2000 y a través del método de línea de pobreza, revelan que para ese año la entidad concentraba 55.6 por ciento de hogares en esa situación contra $\mathbf{6 6 . 4}$ por ciento del entorno nacional.

Y cuarto, el índice de desarrollo humano 8 refleja que la entidad ocupa la tercera mejor posición de bienestar socioeconómico dentro del plano nacional, sólo superada por el Distrito Federal y Nuevo León.

\footnotetext{
8 El índice de desarrollo humano (IDH) es un valioso instrumento de compara ción de las condiciones básicas de vida entre distintas regiones. En esencia, se trata de un indicador compuesto que combina: 1) la longevidad (medida mediante la esperanza de vida al nacer); 2 ) el logro educacional (a través de la alfabetización de adultos y la matrícula combinada de varios niveles educativos); y 3) el nivel de vida, mediante el PIB per capita anual ajustado (paridad del poder adquisitivo en dólares), lo que permite de manera sintética conocer qué región se encuentra en mejor o peor situación socioeconómica. Al incluir los logros en los tres campos indicados, el IDH ha logrado abrir $\mathbf{l}$ abanico de indicadores que pueden utilizarse en la medición del desarrollo, al tiempo que busca medir el progreso socioeconómico de casi todos los páses del mundo a través de unos cuantos indicadores suficientemente homogéneos y relativamente universales.
} 


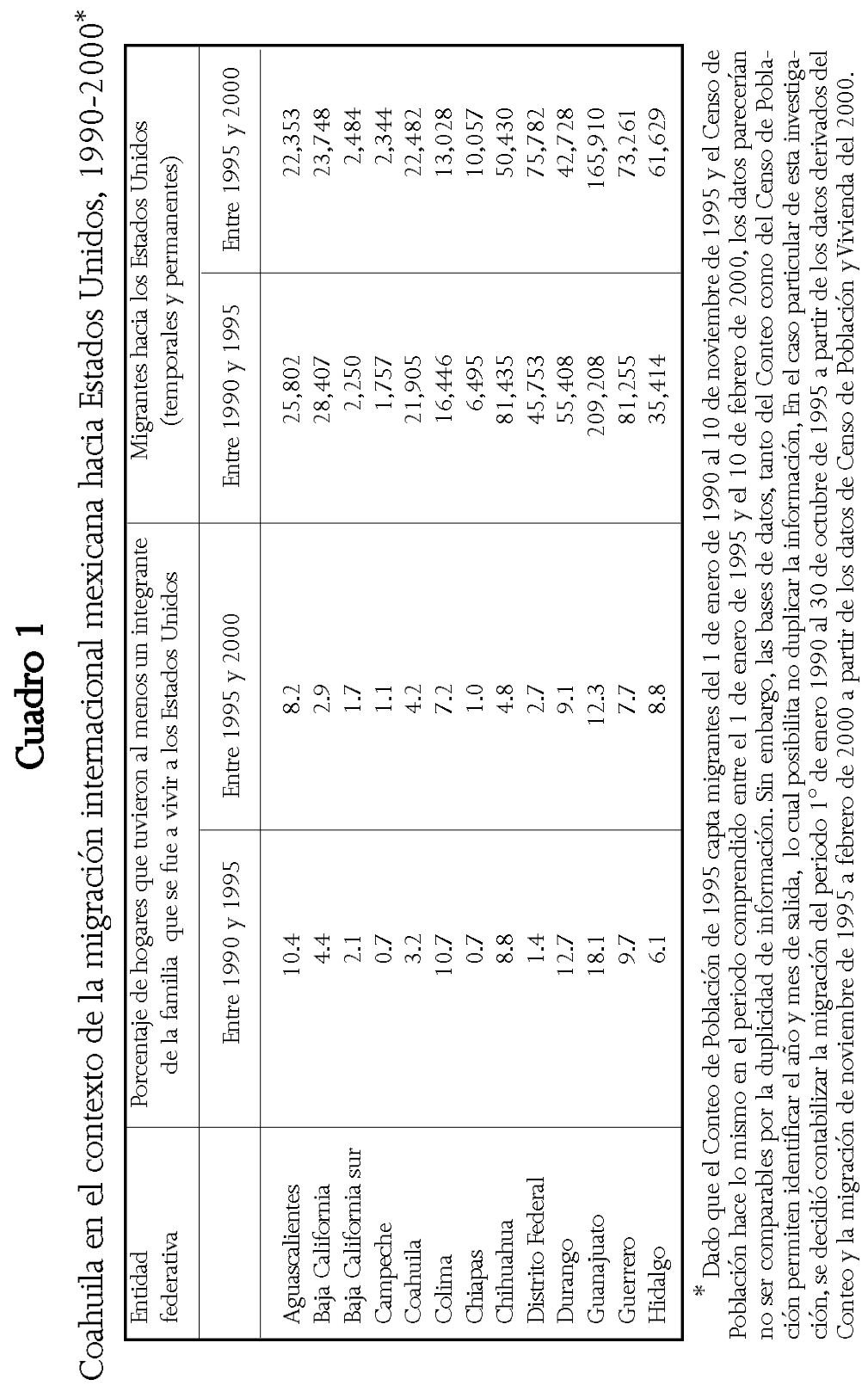




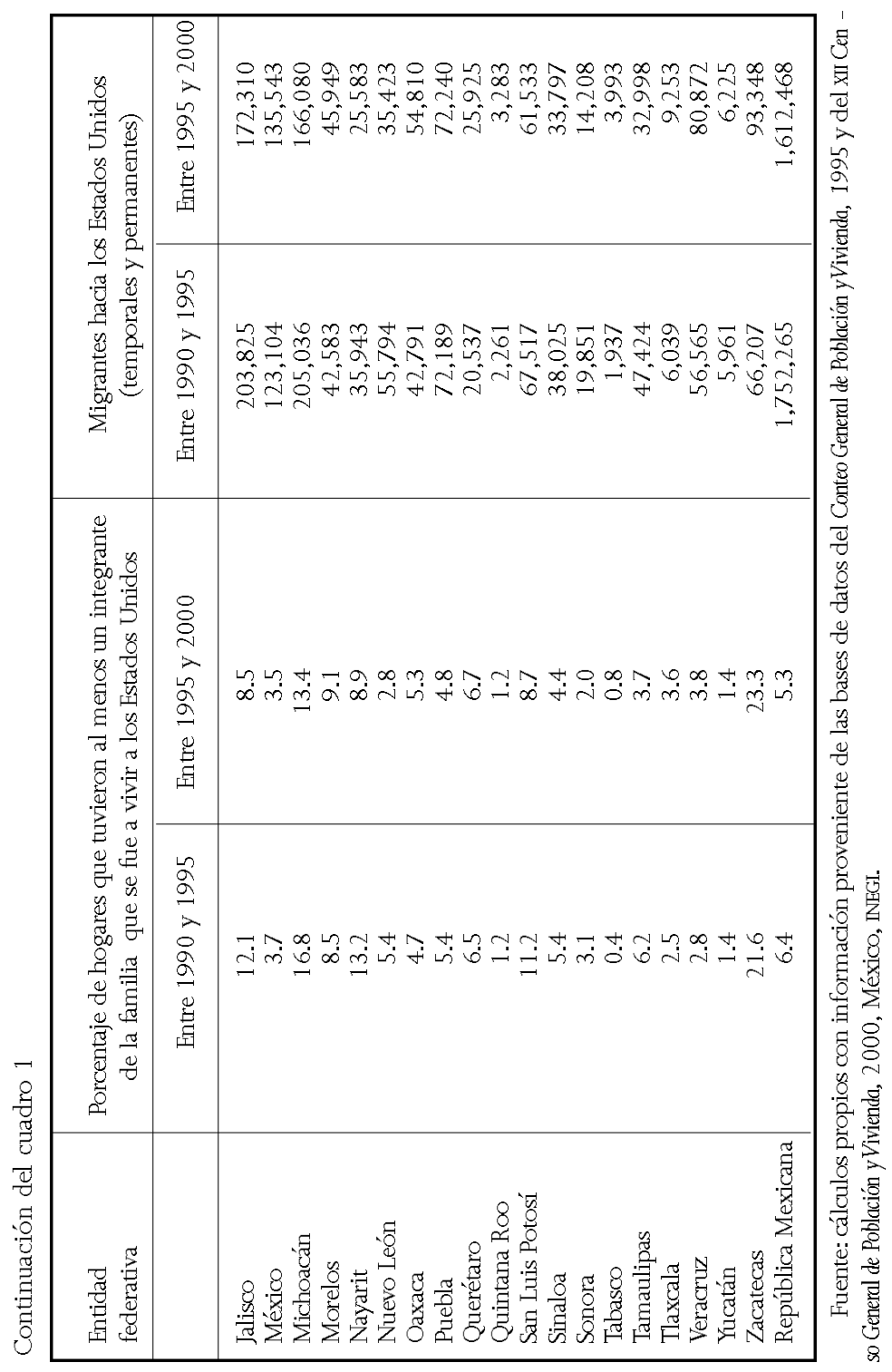


De manera particular, los datos estadísticos referentes al proceso migratorio permiten señalar los siguientes aspectos:

1. A lo largo de la década pasada, poco más de 44 mil coahuilenses se integraron al flujo de connacionales hacia los Estados Unidos, ya sea en forma permanente o temporal. Este dato es equivalente a 1.3 por ciento del total nacional.

2. En Coahuila, sólo 3.6 por ciento del total de hogares contó con la participación de al menos un migrante hacia el vecino del norte en el periodo de referencia. Esta cifra, que se encuentra $\mathbf{5 0}$ por ciento por abajo del promedio nacional, ubica a la entidad como uno de los estados con mediana incidencia dentro del concierto de la migración internacional de mexicanos hacia los Estados Unidos.

3. Aunque sea en términos poco significativos, el fenómeno de la migración internacional dentro del estado ha venido adquiriendo un mayor dinamismo. La comparación entre lo acontecido en los años 1990-1995 y 1995-2000 permite observar que, tanto por el número de migrantes como por el número de hogares con esa característica, la entidad muestra una ma yor intensidad migratoria a lo largo del segundo periodo.

Más allá de estas cifras, que de suyo sirven para ubicar a la entidad en el contexto de la migración internacional, revelan de igual forma la ya secular relación migratoria entre ambos países, configurada por tendencias de marcada continuidad, pero también de significativas fuerzas de cambio; tal es el hecho de la creciente diversificación regional del flujo. Como se observa en los datos del cuadro 1, el origen geográfico de los migrantes se ha extendido más allá de las entidades tradicionales de emigración, y actualmente entidades como Puebla, Hidalgo, Estado de México, Chihuahua y Morelos - por mencionar algunas - así como el Distrito Federal, participan con cuantiosas corrientes migratorias a vecino país.

Esto no significa que el flujo en las entidades tradicionales ha ya disminuido, sino por el contrario, con las tendencias recientes 
se hace más evidente su histórica vocación migratoria. Por ejemplo, los estados que tradicionalmente se han caracterizado por ser fuertes expulsores de población hacia los Estados Unidos concentran los más altos porcentajes de hogares con migrantes (pueden verse los ejemplos de Aguascalientes, San Luis Potosí, Jalisco, Durango, Nayarit, Michoacán, Guanajuato y Zacatecas).

Rasgos distintivos de la migración intemacional en Coahuila

En los últimos años, uno de los aspectos más evidentes que ha caracterizado a la migración de mexicanos hacia los Estados Unidos es la modificación de su tradicional patrón migratorio hacia otro, más complejo y heterogéneo (con volúmenes cuantiosos y crecientes), cuyos rasgos centrales son: 1) el desgaste del patrón circular migratorio en el que es evidente una estancia más larga de los migrantes en el vecino país del norte;2) la decisión de un número cada vez más significativo de mexicanos por establecer su residencia permanente en los Estados Unidos; 3) la creciente diversificación geográfica del lugar de origen de los migrantes, así como de sus características socioeconómicas, y 4) una presencia cada vez más notoria de la mujer en el flujo migratorio (CONAPO, 2000:2-3).

Los datos estadísticos derivados tanto del Conteo de Población yVivienda de 1995 como del XII Censo General de Población y Vivienda del año 2000, permiten derivar importantes referencias empíricas en relación con lo antes descrito: ${ }^{9}$

1. A nivel nacional, de los 1.7 millones de mexicanos que emigraron hacia los Estados Unidos, entre los años de 1990 y 1995, solamente 21.5 por ciento lo hizo de forma temporal,

${ }^{9}$ Las cifras que se presentan a continuación son cálculos propios derivados de las bases de datos de las fuentes antes señaladas. 
mientras que en el periodo 1995-2000 ese porcentaje se re dujo a $17.4 \%$.

2. La mujer ha venido ganando terreno en el flujo migratorio, pues en el primer periodo (1990-1995) representó 25.6 por ciento del total de desplazamientos, en tanto que para los años comprendidos entre 1995 y 2000 participó con poco más de $30 \%$.

3. En 1995, tan sólo seis entidades del país. Guanajuato, Michoa cán, Jalisco, San Luis Potosí,Zacatecas y Durango, concentraban 56.5 por ciento del total de los migrantes hacia los Estados Unidos. Para el año 2000, estas mismas entidades agrupaban sola mente 46.1 por ciento del flujo migratorio.

El tiempo de estancia en los Estados Unidos de los migrantes de retorno se ha incrementado significativamente: hacia 1995, el promedio de estancia de los migrantes en el vecino del norte era 13 meses, mientras que para el año 2000, este indicador alcanza ba la cifra de 15 meses.

En el contorno estatal, hay algunos rasgos que le dan el sello distintivo ala migración de coahuilenses hacia los Estados Unidos y que, sin duda alguna, marcan algunas divergencias y convergencias respecto al patrón migratorio que se viene manifestando en el ámbito nacional.

Espacialmente, la migración estatal hacia el vecino del norte es un proceso que se encuentra concentrado en el área centro-norte del estado y en una pequeña área ubicada al suroeste de la entidad. La primera región, integrada por los municipios de Escobedo, Abasolo, Juárez, Progreso, Sabinas, Villa Unión, Nava,Morelos, Múzquiz,Acuña,Jiménez, San BuenaVentura, Piedras Negras, Castaños, Zaragoza, Monclova y San Juan de Sabinas, se caracteriza por presentar la mayor intensidad del fenómeno migratorio, pues el porcentaje de hogares con migrantes entre 1995 y 2000 oscila entre el 5.3 y 29.8 por ciento.

Incluso, los cuatro primeros municipios manifiestan un patrón muy similar al de las entidades con mayor tradición migratoria, 
como pueden ser Zacatecas, Guanajuato, Michoacán, Jalisco 0 Durango.

Desde una perspectiva económica, esta zona de alta intensidad migratoria se caracteriza por mostrar una alta vocación hacia las actividades industriales. Dos datos son relevantes al respecto: primero, para el año 2000, del total de la población ocupada en esta región, 4.6 por ciento laboraba en el sector agropecuario; 48.7 en el industrial y 46.7 en los servicios. Estos datos marcan algunas diferencias con el contexto estatal, pues la distribución porcentual de la población ocupada en términos de los sectores agropecuario, industrial y de servicios es de 6.1, 41.4 y 52.5 por ciento, respectivamente. ${ }^{10} \mathrm{Y}$ segundo, la región se distingue por concentrar un alto número de unidades económicas dedicadas a la producción industrial, entre las que destaca de manera especial el acero, el carbón y las empresas maquiladoras.

A manera de hipótesis - dado que este trabajo es una primera aproximación al fenómeno de la migración de coahuilenses hacia los Estados Unidos y como tal genera más dudas que respuestas- puede señalarse que, aunque parezca contradictorio, es quizás esta misma estructura económica (o más bien su dinámica) la que esté influyendo en el intenso flujo migratorio que registra la zona. Cabe recordar que, si bien la migración de mexicanos a Estados Unidos es un proceso dinámico en el que interactúan una diversidad de factores históricos, económicos, sociales y culturales, los que actualmente ejercen una mayor influencia en la corriente migratoria son los factores de demanda-atracción en Estados Unidos, los factores de ofertaexpulsión en México y, en menor medida, las redes sociales y familiares que vinculan la oferta y la demanda. Dentro de estos aspectos, destaca de manera particular, la dinámica y calidad del empleo en términos de retribución salarial, prestaciones sociales y estabilidad laboral.

En este sentido, no es de dudarse que la difícil situación económica por la que atraviesan desde hace ya varios años las indus-

\footnotetext{
10 Cálculos propios con datos del XII Censo General de Población y Vivienda del año 2000, México, INEGI.
} 


\section{Cuadro 2}

\section{Localización geográfica de la migración internacional} en Coahuila, 2000

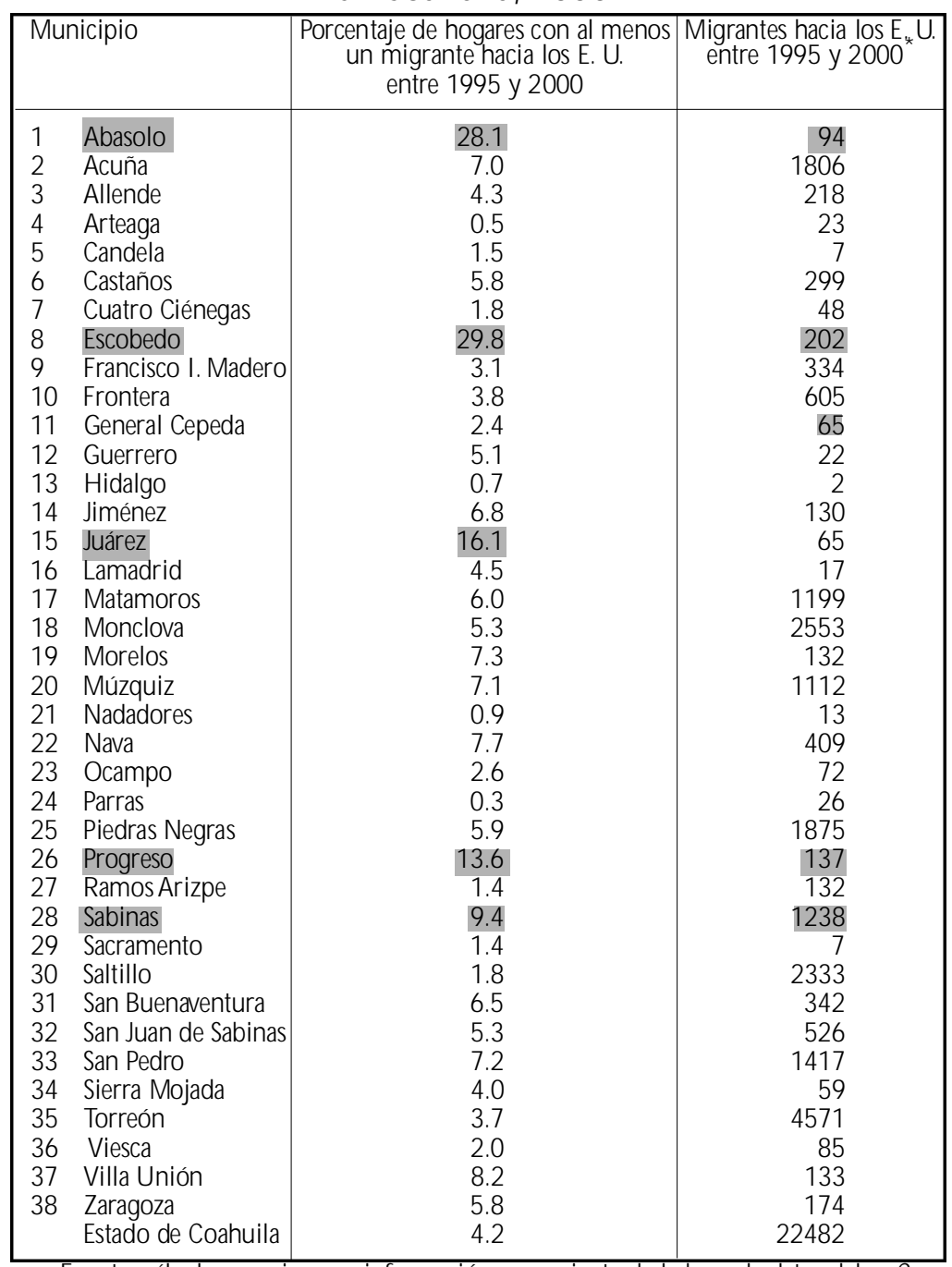

Fuente: cáculos propios con información proveniente de la base de datos de XII Censo General de Población y Vivienda, 2000,México, INEGI.

* Aunque existen municipios que registran un número de casos bastante pequeño, se decidió presentar la información desglosada por municipio y no trabajar por regiones, da do que el presente trabajo busca ser un primer diagnóstico de la migración en Coahuila. 


\section{Mapa}

\section{Localización geográfica de las zonas más importantes de migración hacia los Estados Unidos}

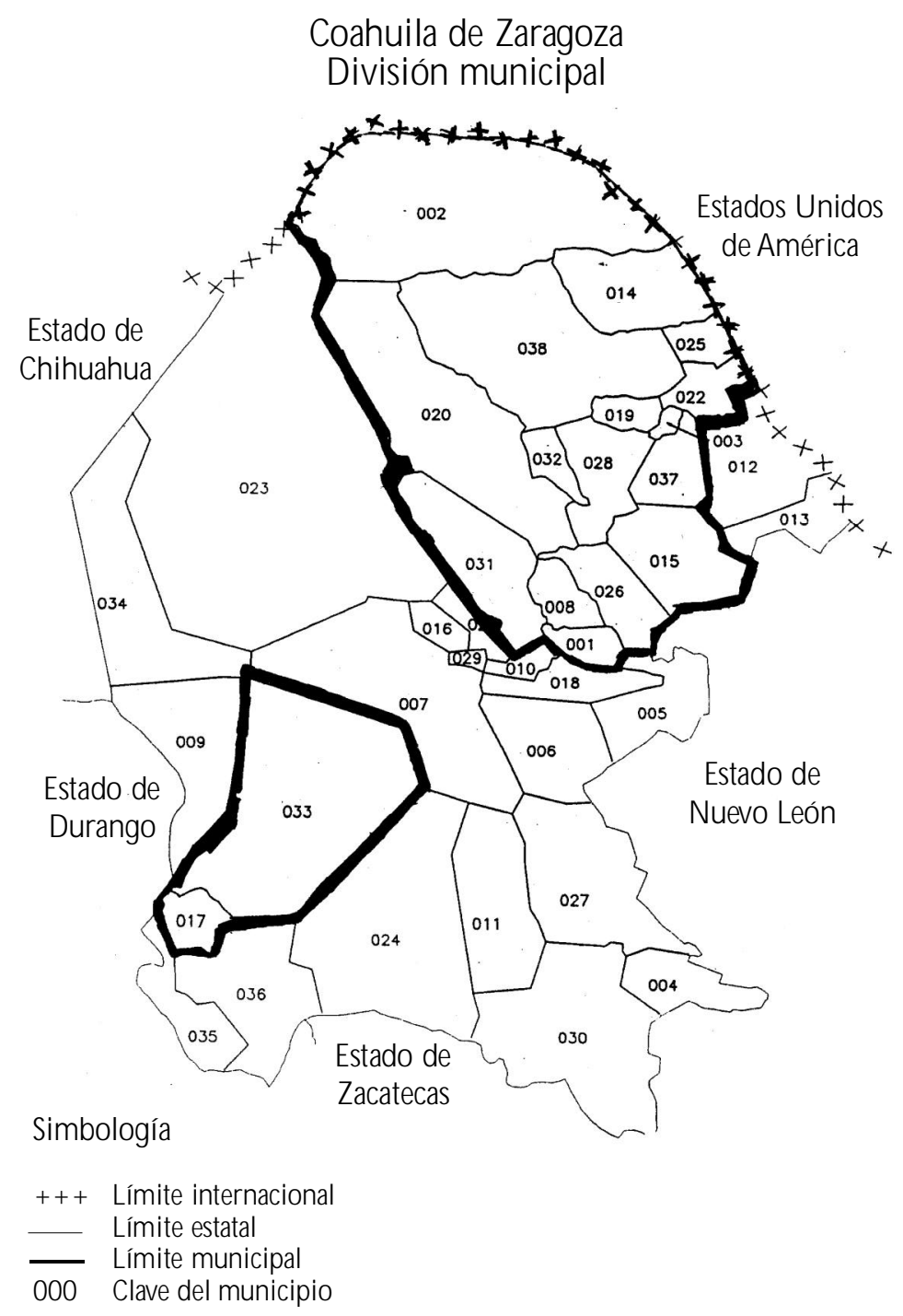


trias del acero y del carbón - en términos de recorte de personal, reducción de prestaciones sociales, disminución e incluso estanca miento en el ritmo de crecimiento de la actividad- se haya convertido en catalizador del fenómeno migratorio hacia los Estados Unidos.

Por su parte, la segunda región de alta intensidad migratoria - localizada en el suroeste del estado- comprende los municipios de San Pedro y Matamoros; su rasgo central distintivo es su alta orientación económica hacia las actividades agropecuarias. Para el año 2000, el 20 por ciento de la población ocupada lo hacía en el sector agropecuario; este porcentaje es tres veces ma yor que el registrado por el contexto estatal. De igual forma que en el caso de la región anterior, se puede considerar como hipótesis que la difícil situación por la que atraviesa el campo mexicano, producto de múltiples factores, tales como los cambios climáticos, la indiscriminada apertura comercial, la descapitalización del sector, los escasos apoyos oficiales y la escasa dinámica en la genera ción de empleos en este tipo de zonas, entre otros, son elementos que pueden estar influyendo en la intensidad del fenómeno migratorio.

Al analizar los rasgos que configuran el patrón migratorio internacional de los coahuilenses hacia los Estados Unidos, destacan los seis siguientes aspectos:

1. U na presencia creciente y mayoritaria de la emigración perma nente. Para 1995, ésta representó 67.6 por ciento del total de los flujos y para el año 2000, se ubicó en $\mathbf{7 3 . 2}$ por ciento.

2. Aunque la emigración temporal representa un menor flujo, sus valores se encuentran por encima del promedio nacional.

3. Al igual que en el panorama nacional, los tiempos de estancia de los migrantes temporales han venido incrementándose: durante el periodo 1990-1995, un migrante temporal se queda ba ocho meses en promedio en los Estados Unidos, en tanto que para los años 1995-2000, su estancia promedio ascendió a 12 meses. Quizás tal incremento refleja los cambios que se están operando en el espectro ocupacional de los trabajadores 
mexicanos en los Estados Unidos, donde las ocupaciones de los migrantes en actividades agrícolas estacionales han perdido importancia relativa frente a otra clase de empleos, y también $\mathrm{el}$ incremento en los costos y riesgos asociados a la migración.

4. Una fuerte presencia femenina en el flujo migratorio, la cual ha permanecido constante a lo largo de la década pasada en el orden del 30 por ciento.

5. Aunque la presencia del varón jefe de familia sigue siendo dominante en el contexto de la migración internacional coa huilense, en la última década se advierte un incremento en la participación de los hijos(as), cuyo peso proporcional crece de 17.3 a 25.5 por ciento en el periodo referido.

6. Muy relacionado con lo anterior, en el mismo periodo disminuye la edad promedio de los migrantes coahuilenses: en los años 1990-1995, este indicador alcanzó un valor de 28.6 años, mientras que para el siguiente periodo, descendió a 25.4 años. Cabe aquí mencionar que poco más de 90 por ciento de los migrantes se ubican en un rango de edad productivo (de 12 a 55 años). ${ }^{11}$

\section{La funcionalidad económica de la migración intemacional para los coahuilenses}

Sin lugar a dudas, las remesas constituyen uno de los beneficios más evidentes de la migración, tanto en términos macroeconómicos como en los ámbitos local y familiar. A nivel agregado, las remesas se han convertido en un flujo de divisas de suma importancia para la mayoría de las naciones no desarrolladas y expulsoras de mano de obra, al punto que en muchos casos constituyen uno de los principales rubros en el renglón de transferencias corrientes de sus balanzas de pagos (Durand, Parrado y Massey, 1996).

\footnotetext{
11 Los datos correspondientes al contexto nacional reflejan que el promedio de edad de los migrantes descendió de 25.4 a 25.1 entre 1995 y 2000.
} 


\begin{tabular}{|c|c|c|c|c|}
\hline \multirow[t]{2}{*}{ Año } & \multicolumn{4}{|c|}{ Sector de origen } \\
\hline & Remesas & Turismo & Petróleo & Agropecuario \\
\hline 1991 & 2,660 & 4,340 & 8,166 & 2,373 \\
\hline 1992 & 3,070 & 4,471 & 8,307 & 2,112 \\
\hline 1993 & 3,333 & 4,564 & 7,418 & 2,504 \\
\hline 1994 & 3,475 & 4,855 & 7,445 & 2,678 \\
\hline 1995 & 3,673 & 4,688 & 8,423 & 4,016 \\
\hline 1996 & 4,224 & 5,287 & 11,654 & 3,592 \\
\hline 1997 & 4,865 & 5,748 & 11,323 & 3,828 \\
\hline 1998 & 5,627 & 6,038 & 7,134 & 3,796 \\
\hline 1999 & 5,910 & 5,907 & 9,920 & 4,144 \\
\hline 2000 & 6,572 & 5,816 & 16,382 & 4,217 \\
\hline 2001 & 8,723 & 5,941 & 12,798 & 3,903 \\
\hline 2002 & 9,815 & 5,958 & 12,798 & 3,903 \\
\hline
\end{tabular}

Fuente:elaborado con datos del InformeAnual de Banco de M éxico, México, 2002 e INEGI, Indicadores Económicos, México, 2001.

En el caso particular de nuestro país, en correspondencia con la intensidad que acusa la migración internacional, el flujo de divisas que generan las remesas provenientes de los Estados Unidos ha venido creciendo con singular dinamismo. En 2002, este flujo alcanzó un máximo histórico cercano a los diez mil millones de dólares (véase cuadro 3). No está por demás agregar que México sobresale, con mucho, como el principal país receptor de remesas familiares en América Latina y el segundo a nivel mundial,después de la India (Lozano, 2000:160-161; Torres, 1998:7-8).

Con ello, la exportación de fuerza de trabajo logra situarse como la segunda fuente neta de divisas en importancia del país, con una contribución a la balanza de pagos muy superior a la correspondiente al turismo.

Más todavía, tomando en consideración su tendencia, las remesas se muestran como la fuente de divisas que registra el creci- 
miento más consistente a lo largo de la década de los noventa. En contraste con otros rubros de exportación, en el caso de las remesas - donde la mercancía que se vende es directamente la fuerza de trabajo- se evidencia la absoluta incapacidad estructural del aparato productivo nacional para generar empleo (Delgado y Rodríguez, 2002).

Este punto se refuerza al considerar el trabajo de Martin (2001:23), quien señala que en el año 2000, laboraban en Esta dos Unidos entre 4 y 5 millones de mexicanos, cifra equivalente a una tercera parte de los trabajadores empleados en el sector formal del pás (según los registros del Instituto Mexicano del Seguro Social) o una quinta parte del total de la población asalariada "ocupada" consignada por el Instituto Nacional de Estadística, Geografía e Informática (INEGI) de México.

En el plano regional, los beneficios de las remesas están concentrados en unas cuantas regiones. Específicamente, las zonas de emigración tradicional (Jalisco, Michoacán,Guanajuato, Zacatecas, Durango, San Luis Potosí, Colima y Aguascalientes) y del norte del país son las que reciben la mayor parte (más de 64\%) del flujo total de remesas Un poco más de 53 por ciento de las remesas transferidas por los migrantes tuvieron como destino la región tradicional y alrededor de 10 por ciento la región norte (cONAPO, 2002a). Además, los impactos regionales y locales de las remesas son sumamente significativos, aunque diferenciados, aun entre los estados que integran la región tradicional de la migración interna cional hacia Estados Unidos. Por ejemplo, se estima que Michoa cán, la entidad que mayores recursos recibe por esta vía, absorbió poco más de 1, 400 millones de dólares por remesas en 2001, en tanto que Zacatecas, undécimo lugar en este rubro, obtuvo alrededor de 277 millones (CONAPO, 2002b).

Sin embargo, más allá de las comparaciones que pudieran ha cerse de las remesas, tanto en términos macroeconómicos como espaciales, hay que valorar la funcionalidad económica que tiene la migración internacional de mexicanos hacia los Estados U nidos en el seno de los hogares (Rodríguez, 1999:140-141). En el caso 


\section{Cuadro 4}

Coahuila: hogares que reciben remesas

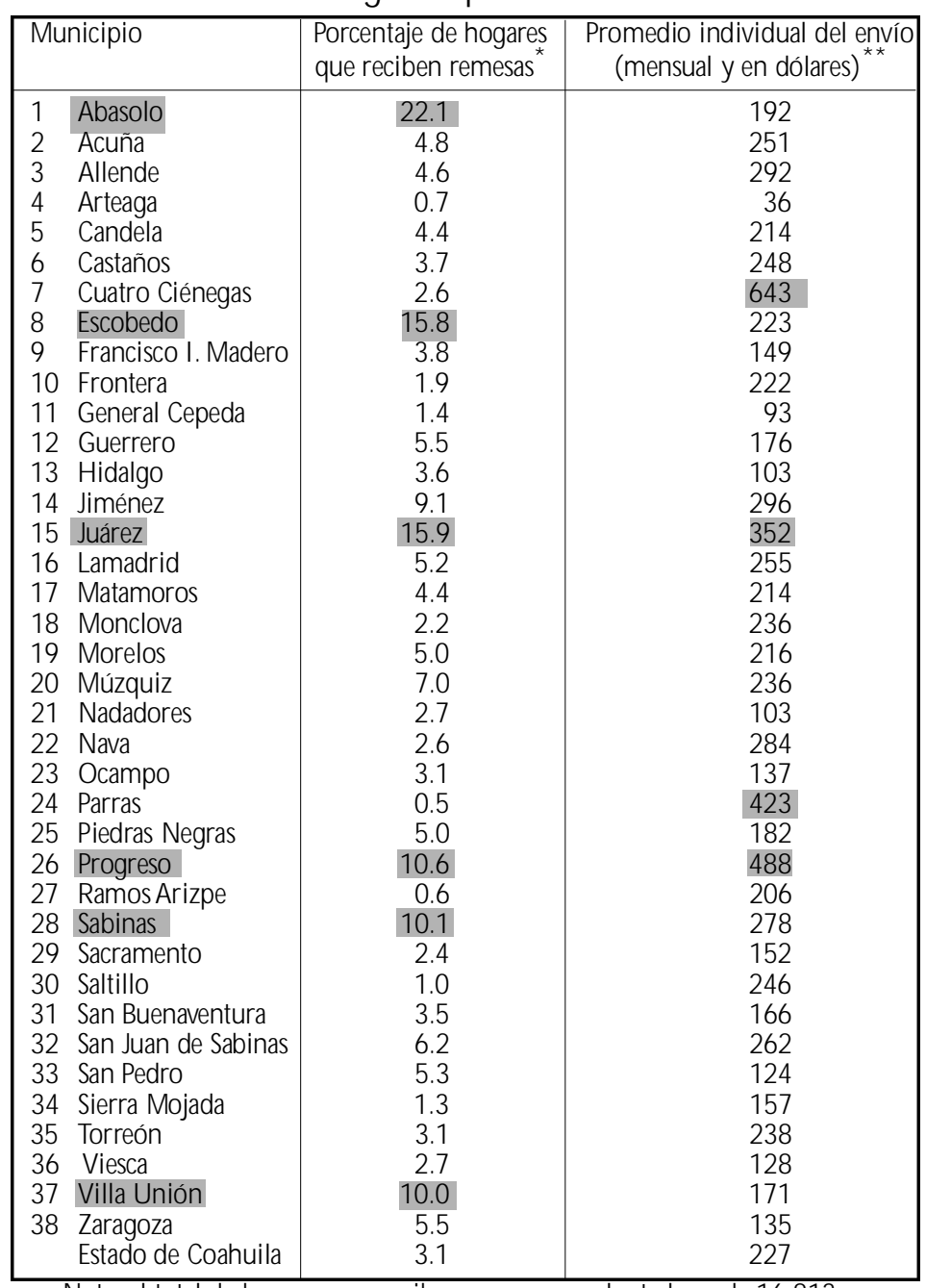

Nota: el total de hogares que reciben remesas en el estado es de 16,813.

La información se refiere al último año; esto es, de febrero de 1999 a febrero de 2000 .

Aunque la encuesta recoge la información en pesos, los datos se convirtieron a dólares utilizando el promedio del tipo de cambio en ventanilla a la compra durante el periodo de febrero de 1999 a febrero de 2000.

Fuente: cálculos propios con información proveniente de la base de datos del XII Censo General de Pobladón y Vivienda, 2000,México, INEGI. 
particular que nos ocupa - la migración de coahuilenses hacia los Estados Unidos-, se ha optado por analizar tres aspectos que permiten ponderar lo antes referido: el primero hace referencia al número de hogares que reciben remesas; el segundo, busca ubicar el peso proporcional que tienen las remesas dentro del ingreso familiar $y$, finalmente, el tercero examina el papel que desempeña este flujo de divisas en la disminución de los niveles de pobreza.

Referente al primer aspecto, se puede señalar que tanto el fenómeno migratorio como las remesas constituyen aspectos generalizados en la vida del país, pues involucran a uno de cada cinco hogares mexicanos, tal proporción se eleva en algunas regiones, como las áreas rurales de nueve entidades federativas del centrooccidente de la República, donde de cada dos hogares uno está relacionado con el vecino país del norte por recibir dólares, porque entre sus miembros hay alguno 0 algunos que vivieron 0 trabajaron (o trabajan) en Estados Unidos o porque de esta unidad doméstica salió alguna persona para radicar en Norteamérica (Corona, 2000:187-189). Coahuila no es ajeno a este proceso, ya que la información censal - derivada del Conteo de Pobla ción y Vivienda de 1995 y del xII Censo General de Población y Vivienda- revela un significativo incremento del porcentaje de hogares que reciben remesas, al pasar de 1.3 a 3.1 por ciento entre 1995 y 2000.

Más aún, los datos estadísticos contenidos en el cuadro anterior hacen evidente la importancia económica de la migración en un considerable número de hogares coahuilenses. ${ }^{12}$ Destaca de

\footnotetext{
12 Cabe señalar que al tratar de medir las remesas familiares vía los censos de población existen algunos problemas que tienden a subestimar su monto real. Corona (2000:178) sintetiza estas limitantes en cuatro aspectos: 1) no se incluye el valor de las remesas en especie; 2) la posible no incorporación de las remesas enviadas por miembros ausentes durante el levantamiento de la encuesta y que el entrevistado considere poco relevantes; 3) la omisión del ingreso obtenido como prestaciones por el trabajo desarrollado en Estados Unidos y 4) no se realiza la captación de las remesas que traen consigo los propios migrantes a su regreso al hogar. Esta situación es especialmente importante cuando se trata de migrantes que viven cerca de Estados Unidos
} 
manera singular el porcentaje de familias que reciben remesas en los municipios de Abasolo, Escobedo, Juárez, Progreso, Sabinas y Villa Unión, pues en todos ellos como mínimo uno de cada 10 hogares recibe recursos provenientes del vecino del norte. Un aspecto que llama la atención es el monto promedio de remesas enviadas y que en muchos casos representa una considera ble cantidad de dinero, como lo evidencian los municipios de Cuatro Ciénegas, Juárez y Progreso. En el contexto nacional, 1.2 millones de hogares se benefician de este flujo de dinero y el promedio mensual de ingreso por concepto de remesas ascendió a poco más de 190 dólares; por ello, la entidad se ubica muy por encima de la tendencia nacional.

Otro ángulo, desde el que puede verse la funcionalidad económica de las remesas es mediante el análisis de su importancia dentro del ingreso del hogar. Al respecto, un estudio realizado por el conAPO (2002) con datos de la Encuesta Nacional de Ingreso Gasto para el año de 1998, muestra que a nivel nacional las remesas representan en los hogares que las reciben poco menos de la mitad (41\%) de su ingreso total; 44 por ciento en localidades con 2500 o más habitantes, y 50 por ciento en los hogares situados en localidades con menos de 2500 habitantes. En Coahuila, casi $17 \mathrm{mil}$ hogares reciben remesas cuya importancia, al igual que en el plano nacional, queda de manifiesto al considerar el hecho de que en promedio representan 50 por ciento del ingreso total del hogar (véase cuadro 5). Más todavía, al profundizar en el análisis puede corroborarse el papel que esta fuente de ingreso cumple dentro de los hogares, ya que en uno de cada cuatro que reciben remesas, éstas constituyen la única fuente de ingresos. En algunos municipios, este indicador se aproxima a cerca del 50 por ciento.

Finalmente, un tercer aspecto que permite dimensionar la funcionalidad económica de este flujo de divisas en los hogares es $\mathrm{U}$ análisis de los niveles de pobreza entre hogares receptores y

y que realizan frecuentemente los traslados laborales a ese país, como puede ser el caso de Coahuila. 


\section{Cuadro 5}

\section{Coahuila: Importancia de las remesas en el ingreso de los hogares que reciben remesas}

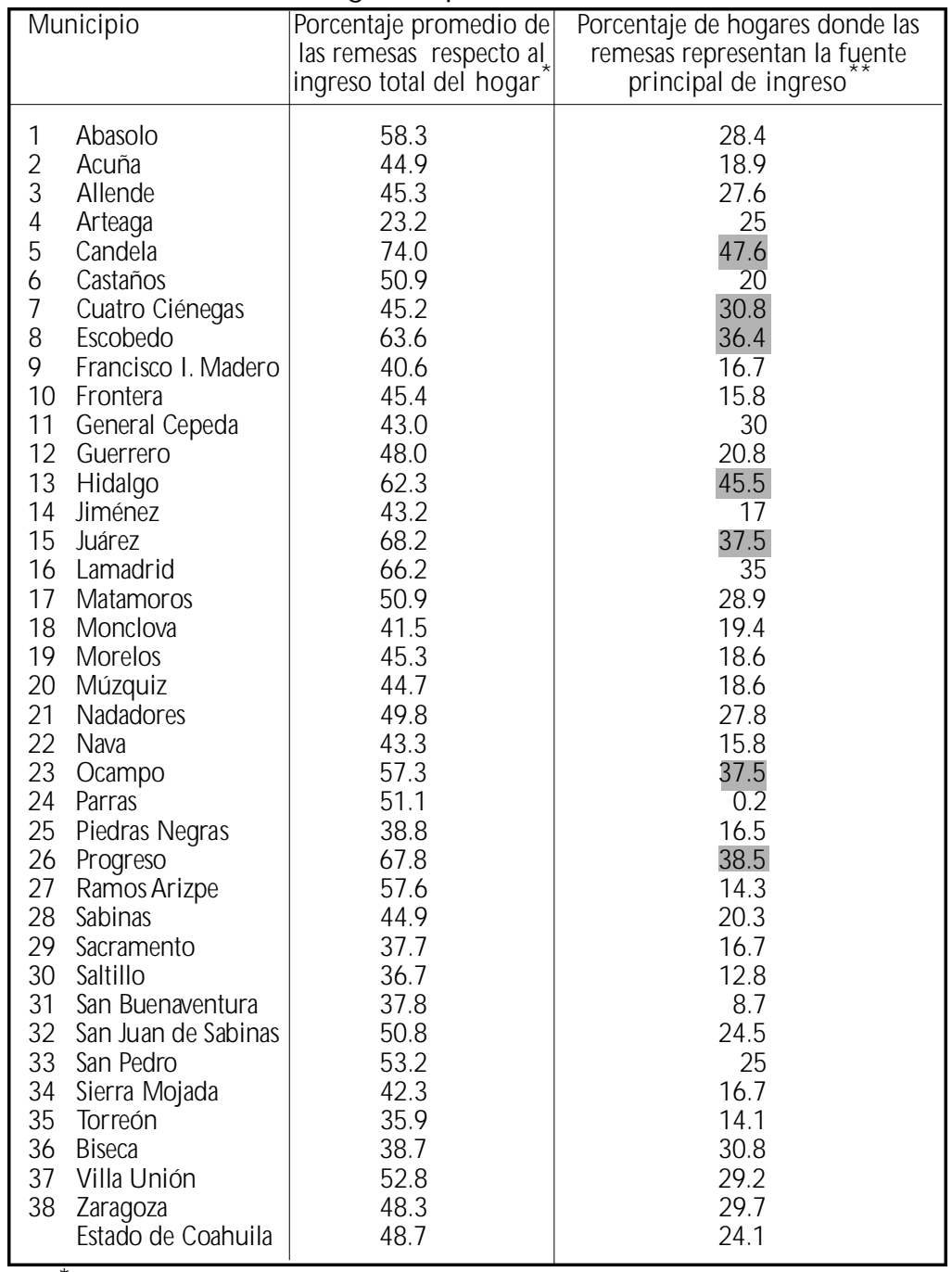

* En ambos casos $s$ refiere sólo a hogares que reciben remesas.

** Por fuente principal de ingreso se entiende aquella situación en la que las remesas representan más del 90 por ciento del ingreso total del hogar.

Fuente: cálculos propios con información proveniente de la base de datos del XII Censo General de Población y Vivienda, 2000,México, INEGI. 
no receptores de remesas. En el campo de los estudios de migra ción este tema ha sido el centro de importantes debates; en términos generales se ha logrado consenso en torno a que la emigración hacia los Estados Unidos es ante todo una estrategia de los hogares para mejorar sus ingresos y un medio eficaz para alcanzar el objetivo de mejorar sus condiciones de vida (Marceli, 2001; CONAPO, 2002a).

S bien en el plano macroeconómico las remesas constituyen un importante paliativo para compensar los fuertes desequilibrios que en términos de la balanza comercial enfrenta nuestro país, 13 los datos estadísticos contenidos en el cuadro 6 permiten señalar un mayor impacto a nivel microeconómico. En efecto, la compa ración de los niveles de pobreza entre hogares que reciben remesas y aquellos que no se benefician de este recurso (columnas B y C del cuadro referido) permiten valorar el papel que las remesas están cumpliendo como paliativo en la disminución de los alarmantes niveles de pobreza que hoy en día padecen miles de familias coahuilenses.

Las estimaciones de pobreza realizadas en este trabajo - con información proveniente del xII Censo General de Población y Vivienda del año 2000 y bajo el método de línea de pobrezaindican que de los $560 \mathrm{mil}$ hogares que conforman a la entidad para el año 2000 (reciban o no remesas), poco más de una tercera parte (35.7\%) se encuentran en una situación de pobreza de patrimonio (recordemos que este tipo de pobreza hace referencia a aquella situación en la que el ingreso total del hogar es menor al necesario para cubrir el patrón de consumo básico de alimentación, vestido, calzado, vivienda, salud, transporte público y educación, de todos los miembros del hogar). Si comparamos este resultado con el porcentaje de hogares que reciben remesas y resultaron ser pobres, claramente se observa una tendencia significativa a la disminución de este lacerante mal social: en prome-

\footnotetext{
13 Se estima que para el año 2002, el déficit comercial de México ascendió a poco más de 9 mil millones de dólares.
} 


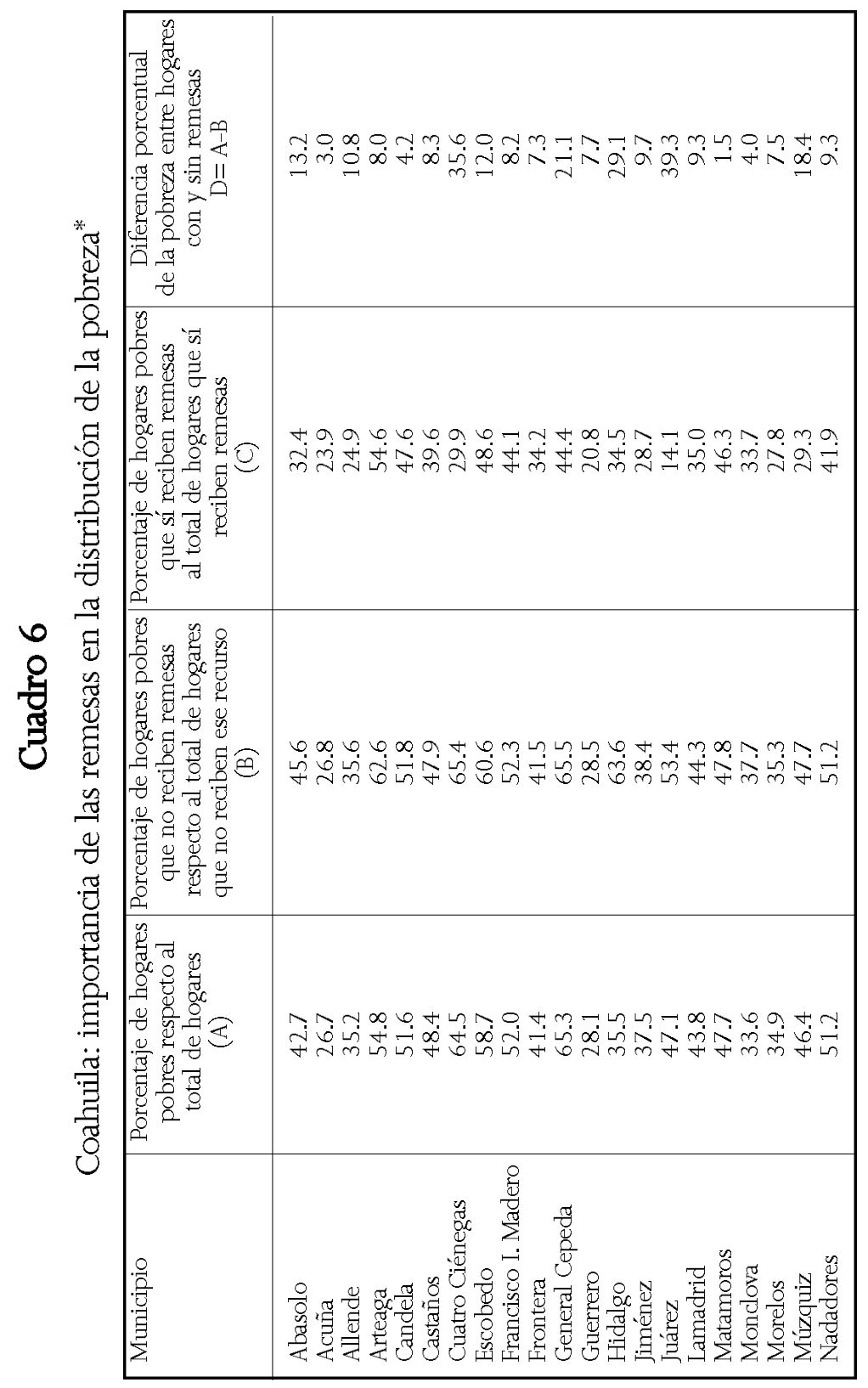




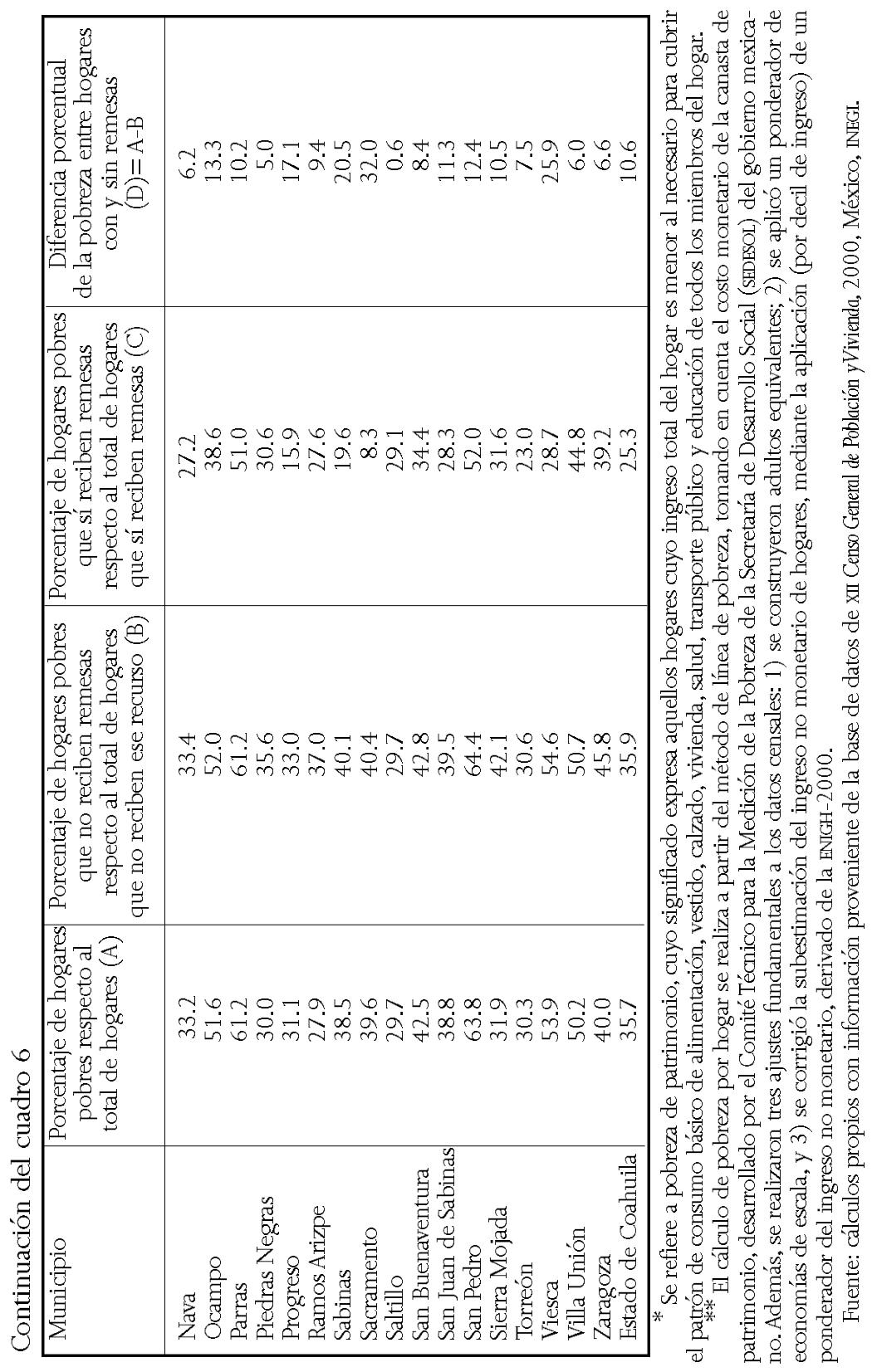


dio sólo uno de cada cuatro hogares que reciben remesas pueden ser clasificados como pobres. Resultados que muestran la significativa incidencia de las remesas en la disminución de la pobreza.

Más aún, si en Coahuila los 17 mil hogares que reciben remesas dejaran de percibirlas, el número de hogares pobres en la entidad se incrementaría en casi 10 mil familias y el número de coahuilenses que se integraría a las filas de la pobreza sería de poco más de 50 mil personas.

Cabe resaltar que las remesas llegan directamentea los hogares de los familiares de los migrantes y cumplen un papel determinante en el sostenimiento familiar. Su impacto en las comunida des y en los hogares receptores a menudo se pone de manifiesto a través de la información relativa al monto y modalidades de uso de estos recursos. La mayoría de los trabajos disponibles dan cuenta de un patrón general del uso de las remesas en México, congruente con numerosas experiencias internacionales, que indican que la gran mayoría de los recursos recibidos se gastan en la satisfacción denecesidades básicas, en la adquisición de bienes de consumo duradero y en la compra y mejora de vivienda, mientras que sólo una pequeña porción se destina al ahorro y a la inversión productiva (Rodríguez, 1999:135).

\section{Reflexiones finales}

De lo hasta aquí expuesto, es posible identificar las principales tendencias que acusa hoy en día el fenómeno de la migración de coahuilenses hacia el vecino del norte. Se trata de un proceso con amplia convergencia hacia la dinámica mostrada por el contexto nacional. De manera particular, el patrón migratorio de los coahuilenses puede ser descrito por: una fuerte presencia de la migración permanente, con la participación mayoritaria de varones jefes de familia y una significativa participación del sector femenino en el flujo hacia el vecino del norte; todo ello, coronado por la presencia de una abrumadora población joven en edad productiva. 
Entre las pocas diferencias entre el contexto estatal y el panorama nacional es la escasa participación que tienen las ciudades urbanas medias y grandes de la entidad, como trampolín hacialos Estados Unidos.

De igual forma, los hallazgos de esta investigación permiten corroborar la hipótesis central planteada en este trabajo, pues si bien quedó demostrado que en términos cuantitativos la migra ción internacional de coahuilenses hacia los Estados Unidos no es muy significativa como en otras entidades del país, la funcionalidad económica del fenómeno es de suma trascendencia para el sostenimiento de las familias y la reducción de los niveles de pobreza. Las remesas constituyen un recurso económico fundamental para el sostenimiento familiar y de sus comunidades, a la vez que un elemento dinamizador - en algunos casos imprescindible- para ciertos sectores de las economías locales y regionales, como son las ramas de bienes de consumo inmediato, el comercio en general, los servicios y la actividad financiera asociada al cambio de dólares por pesos.

Recibido en octubre de 2002

Revisado en enero de 2003

\section{Bibliogrefía}

Bean, Frank y Martha Tienda (1987), The Hispanic Population of the United States, NuevaYork, Ruseell Sage Foundation.

Bustamante, Jorge A. (1997), Cruzar la líne日. La migración de mexicanos a Estados Unidos, México, Fondo de Cultura Económica (Sección de O bras de Sociología).

CONAPO (2000),"Trabajadores adolescentes y jóvenes en la migra ción internacional hacia los Estados Unidos, 1998-2000", Consejo Nacional de Población, Boleín de Migración Internacional, no. 11. 
(2001), Informe de trabajo sobre la situación actual de la migración internacional de mexicanos hadia los Estados Unidos, México, Consejo Nacional de Población.

(2002a), La migración internacional de mexicanos hacia los Estados Unidos: presente y futuro, México, Consejo Nacional de Población.

(2002b), I mportancia de las remesas en el ingreso de los hogares me xicanos, México, Consejo Nacional de Población.

Corona, Rodolfo (2000), "Monto y uso de las remesas en México", en Rodolfo Tuirán, Migración México-Estados Unidos: opciones de política, México, Consejo Nacional de Población, pp. 167-190.

y Rodolfo Tuirán (1998), "Tamaño y características de la población mexicana en edad ciudadana residente en el país y en el extranjero durante la jornada electoral del año 2000", en IFE, Informe final de la Comisión de Especialistas que estudia las moda lidades del voto de los mexicanos residentes en el extranjero, México, Instituto Federal Electoral, Anexo I, Subcomisión Sociodemográfica, cuadro 2-4.

Delgado, Raúl y Héctor Rodríguez (2000), "Las nuevas tendencias de la migración internacional: el caso de Zacatecas", Comercio Exterior, vol. 50, no. 5, pp. 371-380.

(2002), "El nuevo panorama de la migración interna cional y sus potencialidades para el desarrollo regional", en Jesús Arroyo y Alejandro Canales, El norte de todos: migración y empleo en tiempos de globalización, México, UCLA-Juan Pablos Editores, pp. 116-138.

Durand, Jorge, Emilio Parrado y Douglas Massey (1996), “Migradollars and Development a Reconsideration of the Mexican Case", International Migration Review, vol. 30, no. 2. 
(1998), “¿Nuevas regiones migratorias?”, en René Zenteno (coord.), Población, desarrollo y globalización, México, Sociedad Mexicana de Demografía y El Colegio de la Frontera Norte, pp. 104-106.

, Douglas Massey y René Zenteno (2001), “Mexican Inmigration To United States: Continuities and Changes", Latin American Research Review, vol. 36, no. 1, pp. 107-127.

Escobar, Agustín, Frank D. Bean y Sydney Weintraub (1999), La di námica de la emigradión mexicana, México, CIESAs-Miguel Ángel Porrúa.

Lozano, Fernando (2000), "Experiencias internacionales en el envío y uso de las remesas", en Rodolfo Tuirán, Migradión MéxicoEstados U nidos: opciones de política, México, Consejo Nacional de Población, pp. 191-202.

Marcelli, Enrico (2001), "The Changing Profile of Mexican Migrants to the United States: $\mathrm{N}$ ew Evidence From California and Mexico", Latin American Research Review, vol. 36, no. 3, pp. 105131.

Martin, Philip (2001), "Migration and Development The Mexican-US Case", Simposio sobre migración internadi onal en las Américas, 4 al 6 de septiembre de 2001, Chile, CEPAL.

Rodríguez, Héctor (1999), "Resultados de la encuesta sobre migrantes internacionales en nueve localidades del estado de $\mathrm{Za}$ catecas", en Miguel Moctezuma y Héctor Rodríguez, Impacto de la migración y las remesas en el crecimiento económico regional, México, Se nado de la República, pp.123-145.

Torres, Federico (1998), "Uso productivo de las remesas en EI Salvador, Guatemala, H onduras y Nicaragua", Chile, CEPAL, Documento deTrabajo LC/ MEX/ R.662. 
Tuirán, Rodolfo (2000), M igración M éxico-Estados Uni dos: opci ones de po lítica, México, Consejo Nacional de Población, pp. 191-202. 
